\section{Perfil demográfico, socioeconômico e de saúde de crianças e adolescentes trabalhadores e não trabalhadores, Brasil: análise das desigualdades}

Demographic, socioeconomic, and health profile of working and non-working Brazilian children and adolescents: an analysis of inequalities

\section{Perfil demográfico, socioeconómico y salud de los niños y adolescentes trabajadores y no trabajadores, Brasil: análisis de las desigualdades}

\section{Abstract}

${ }^{1}$ Faculdade de Ciências Médicas, Universidade Estadual de Campinas, Campinas, Brasil.

2 Universidade Federal da Grande Dourados, Dourados, Brasil.

Correspondência I. O. C. Miquilin Departamento de Saúde Coletiva, Faculdade de Ciências Médicas, Universidade Estadual de Campinas.

Rua Tessália Vieira de Camargo 126, Campinas, SP 13083-887, Brasil. isabellaoc@gmail.com

\begin{abstract}
The objectives of this study were to describe the work done by Brazilian children and adolescents and compare the socioeconomic and health profile of those that worked (or were looking for work) versus non-working youngsters. Based on the 2008 Brazilian National Sample Household Survey (PNAD/2008), we selected children and adolescents 5 to 17 years of age, divided into two analytical categories: "workers" (working or looking for employment) and "non-workers". We calculated prevalence rates for the characteristics of their main work, as well as socioeconomic and health variables comparing the two categories. Poisson regression was used to estimate prevalence ratios, adjusted by health characteristics, with "non-workers" as the reference category. Compared to "non-workers", the "workers" category was associated with a higher proportion of boys; age 14 to 17 years; black or brown skin color; lower school attendance; and worse housing conditions. Child labor was associated with worse self-rated health; chronic backache; arthritis or rheumatism; and depression. Effective policies to support families need to be strengthened to effectively fight child labor.
\end{abstract}

Health Surveys; Health Inequalities; Child Labor
Isabella de Oliveira Campos Miquilin 1

Leticia Marín-León 1

Verônica Gronau Luz 2

Ehideé Isabel Gómez La-Rotta 1

Heleno Rodrigues Corrêa Filho ${ }^{1}$

\section{Resumo}

Os objetivos do estudo foram descrever o trabalho de crianças e adolescentes brasileiros e comparar o perfil socioeconômico e de saúde dos que trabalhavam ou buscavam emprego com os não trabalhadores. A partir da Pesquisa Nacional por Amostra de Domicílios (PNAD/2008), selecionamos crianças e adolescentes de 5 a 17 anos divididos em duas categorias de análise: "trabalhadores" (trabalhavam ou buscavam emprego) e "não trabalhadores". Calculamos prevalências das características do trabalho principal, bem como das variáveis socioeconômicas e de saúde entre as categorias de análise. Razões de prevalência ajustadas das características de saúde foram estimadas utilizando-se a regressão de Poisson, sendo "não trabalhadores" a categoria de referência. Comparados aos "não trabalhadores", os "trabalhadores" tiveram maior proporção de meninos; idade entre 14 e 17 anos; pretos e pardos; menor frequência escolar e piores condições de moradia. O trabalho infantil esteve associado à pior saúde referida; doença de coluna ou costas; artrite ou reumatismo e depressão. Políticas eficazes de apoio às famílias devem ser fortalecidas para o efetivo combate ao trabalho infantil.

Inquéritos Epidemiológicos; Desigualdades em Saúde; Trabalho de Menores 


\section{Introdução}

O trabalho é elemento central na vida das pessoas nas sociedades, auxiliando na determinação do acesso a bens e serviços, condições de saúde e bem-estar. Entretanto, a persistência da exploração do trabalho infantil 1 distancia o trabalho de seu papel estruturante, impede a consolidação dos direitos das crianças e adolescentes, perpetua uma estrutura social provedora de desigualdades, além de configurar violação dos direitos humanos. A fragilidade do mercado de trabalho, atribuída às políticas econômicas de precarização das relações de emprego ${ }^{2}$, mantém persistentes as desigualdades sociais entre famílias brasileiras.

Segundo estimativas da Organização Internacional do Trabalho (OIT), 168 milhões de crianças e adolescentes de 5 a 17 anos trabalhavam em 2012 no mundo ${ }^{3}$. No Brasil, o trabalho infantil representa uma realidade para aproximadamente 3,7 milhões de crianças e adolescentes 4 . Apesar de o trabalho infantil ter reduzido $13,4 \%$ na última década 5 , houve aumento de $1,5 \%$ na faixa etária de 10 a 13 anos 6 , idades em que o trabalho não é legalmente aceito no país. O Estatuto da Criança e do Adolescente (ECA; Lei no 8.069/1990) 7 determina a proibição do trabalho a menores de 16 anos, salvo na condição de aprendiz, a partir de 14 anos.

Parte do progresso realizado na redução do trabalho infantil pode ser atribuída a programas sociais como o Programa de Erradicação do Trabalho Infantil (PETI/2011-2015) 8. Como meta, o Brasil pretende alcançar a erradicação do trabalho infantil até 2020, mas a ausência de articulação entre as diversas políticas de combate ao trabalho de crianças e adolescentes, o pouco diálogo entre as esferas do governo e a concessão de autorizações judiciais para o trabalho dificultam o alcance desse objetivo 6,9 .

É preciso considerar que tal objetivo está inserido em uma perspectiva mundial, considerando o conjunto de metas de desenvolvimento sustentável propostas pela Organização das Nações Unidas (ONU) a serem alcançadas até 2030 10. Dentre as 17 metas da agenda de desenvolvimento pós-2015, a oitava proposta estabelece a necessidade de crescimento econômico sustentável e a promoção de trabalho decente, incluindo a urgência de adoção de ações de combate ao trabalho infantil com meta de erradicação até 2025.

Ainda que a experiência brasileira na redução do trabalho infantil seja reconhecida internacionalmente 11 , a persistência do trabalho de crianças e adolescentes em atividades que comprometam a educação formal e a saúde é fonte de preocupação, principalmente pela vulnerabilidade desse grupo à violação de seus direitos. Trata-se de um tema prioritário nas agendas de políticas públicas nacionais. Para a Nova Agenda Global para a Equidade em Saúde, elaborada pela Comissão de Determinantes Sociais da Saúde da Organização Mundial da Saúde (CDSS/ OMS), o trabalho de crianças e adolescentes é influenciado por arranjos políticos e econômicos e considerado um determinante social da saúde 12 .

Causas estruturais como o nível de concentração de renda, precarização das relações de trabalho, desemprego e falta de política educacional integral são fatores associados à inserção da criança no mercado de trabalho. No entanto, devem ser considerados fatores culturais que influenciam a forma pela qual o trabalho infantil é visto, aceito, e, até mesmo, desejável 13.

O trabalho infantil apresenta múltiplas abordagens sobre seu conceito e origem 14 , sendo um tema de preocupação legítimo em Saúde Pública, principalmente pelos danos imediatos e em longo prazo, tais como: perda da infância; exploração econômica pelos baixos pagamentos (quando remunerados); riscos à saúde física $\mathrm{e}$ mental (principalmente por substituírem o trabalho de adultos); perpetuação da pobreza e aumento da probabilidade de se tornarem adultos com menores rendimentos e baixa qualificação profissional devido à entrada precoce no mercado de trabalho 15.

Estudo de revisão 16 identificou maior vulnerabilidade das crianças em relação aos adultos frente aos fatores de risco presentes no ambiente de trabalho. Os autores relatam que foi identificado déficit de crescimento entre crianças que trabalham em áreas rurais e que não frequentavam escola. Outro estudo aponta que a entrada precoce no mercado de trabalho pode aumentar o risco de relatar problemas de saúde na fase adulta 17 .

Além de estarem mais propensas a serem exploradas por longas jornadas de trabalho 18, que por si já é um fator que pode predispor a agravos e adoecimentos, crianças e adolescentes encontram-se em uma etapa de desenvolvimento físico e psicológico que as tornam mais vulneráveis a acidentes e doenças. Deve-se considerar que as consequências do trabalho infantil não são apenas imediatas, uma vez que a exposição a determinados fatores de risco podem gerar doenças que se manifestam apenas na idade adulta.

No Brasil, os dados nacionais sobre trabalho infantil são, em geral, provenientes de inquéritos de abrangência nacional como a Pesquisa Nacional por Amostra de Domicílios (PNAD). Em anos específicos, a pesquisa conduz um suplemento de saúde concomitante à pesquisa sobre traba- 
lho e rendimentos. No entanto, até onde se sabe, apenas estudos utilizando modelos econométricos têm buscado fazer associações entre trabalho infantil e condições de saúde a partir dos dados provenientes da PNAD 19,20.

Ante o exposto, os objetivos do estudo foram descrever, segundo faixa etária, as características do trabalho principal de crianças e adolescentes e comparar o perfil demográfico, socioeconômico e de saúde dos que trabalhavam ou estavam em busca de emprego com os não trabalhadores.

\section{Metodologia}

Trata-se de estudo de análise de dados secundários que utilizou, como fonte, a pesquisa básica e o suplemento de saúde da PNAD de 2008. A PNAD é uma pesquisa conduzida pelo Instituto Brasileiro de Geografia e Estatística (IBGE), realizada por meio de uma amostra probabilística de domicílios obtida em três estágios de seleção: unidades primárias - municípios; unidades secundárias - setores censitários e unidades terciárias - unidades domiciliares 21 . As informações são coletadas mediante entrevistas, sendo utilizado um questionário com questões sobre características gerais da população, educação, trabalho, rendimentos e habitação.

Em 2008, o tamanho da amostra da PNAD foi de 391.868 pessoas selecionadas aleatoriamente em 150.591 unidades domiciliares de áreas urbanas e rurais das 26 Unidades da Federação e do Distrito Federal. Além das questões rotineiramente levantadas, foi introduzido um suplemento de saúde que investigou, dentre outras variáveis, aspectos de morbidade da população 21 .

\section{Sujeitos da pesquisa e variável} independente principal

Selecionamos crianças e adolescentes de 5 a 17 anos de ambos os sexos $(n=91.377)$. Foram consideradas crianças todas as pessoas de até 12 anos de idade incompletos, sendo os adolescentes aqueles entre 12 e 18 anos de idade, conforme o ECA 7 .

A variável independente principal foi o status ocupacional das crianças e adolescentes, dividido em duas categorias de análise:

(a) Trabalhadores: corresponderam à soma das crianças de 5 a 9 anos que exerceram atividades na semana de referência da pesquisa $(n=289)$; das crianças de 5 a 9 anos que exerciam trabalho remunerado na semana de referência mas estavam temporariamente afastadas de suas atividades no período $(n=2)$ e das crianças e adolescentes de 10 a 17 anos economicamente ativos que na semana de referência da pesquisa eram ocupados $(n=8.813)$ ou desocupados $(n=1.898)$. São consideradas pessoas economicamente ativas desocupadas aquelas de 10 anos ou mais de idade que não tinham trabalho na semana de referência da pesquisa, mas que estavam em busca de emprego 21

(b)Não trabalhadores: aquelas que não exerceram qualquer atividade e não foram consideradas pertencentes à população economicamente ativa $(\mathrm{n}=80.375)$.

\section{Variáveis independentes}

(a) Demográficas e socioeconômicas: sexo; faixa etária (5 a 9 anos; 10 a 13 anos; 14 a 17 anos); cor ou raça autorreferida (branca; preta e parda; amarela; indígena); macrorregião de residência (Sudeste; Sul; Centro-oeste; Norte; Nordeste); faixa de rendimento mensal domiciliar per capita em salários mínimos (sem rendimentos; até 1/4; $>1 / 4$ a $1 / 2$; > $1 / 2$ a $1 ;>1$ a 2 ; > 2); "frequenta escola ou creche?" (sim; não).

(b) Características da unidade domiciliar: "domicílio possui água canalizada?" (sim; não); "presença de banheiro ou sanitário no domicílio?" (sim; não); destino do lixo domiciliar (coletado diretamente ou indiretamente; outro - queimado ou enterrado na propriedade, jogado em terreno baldio ou logradouro, jogado em rio, lago ou mar, outro destino); "possui geladeira?" (sim; não); "domicílio cadastrado na unidade de saúde da família?" (sim; não).

(c) Características do trabalho principal de crianças entre 5-9 anos de idade: situação de residência (urbana; rural); atividade principal do empreendimento do trabalho (não agrícola; agrícola); grupamentos de atividade principal do empreendimento (agricultura e pecuária; indústria de transformação; construção; comércio e reparação; alojamento, alimentação; outros - transporte; outras atividades; serviços domésticos; atividades mal definidas); posição na ocupação no trabalho (empregado; trabalhador doméstico; conta própria; não remunerado membro da unidade domiciliar; outro trabalhador não remunerado; trabalhador na produção para o próprio consumo ou na construção para o próprio uso); horas habitualmente trabalhadas por semana no(s) trabalho(s); "cuidava dos afazeres domésticos?” (sim; não); horas dedicadas por semana aos afazeres domésticos.

(d) Características do trabalho principal de crianças e adolescentes de 10 a 17 anos de idade: situação de residência; atividade principal do empreendimento do trabalho (não agrícola; agrícola); posição na ocupação no trabalho agrícola (empregador; empregado permanente; empregado 
temporário; conta própria; não remunerado membro da unidade domiciliar e outro trabalhador não remunerado; trabalhador na produção para o próprio consumo); posição na ocupação no trabalho não agrícola (empregado; trabalhador doméstico; conta própria; empregador; não remunerado membro da unidade domiciliar e outro trabalhador não remunerado; trabalhador na construção para o próprio uso); grupamentos de atividade principal do empreendimento (educação, saúde e serviços sociais; agrícola; outras atividades industriais; indústria de transformação; construção; comércio e reparação; alojamento, alimentação; transporte, armazenagem e comunicação; administração pública; serviços domésticos; outros serviços; outras atividades; atividades mal definidas); rendimento mensal de todos os trabalhos em salários mínimos (sem rendimentos até $1 / 4 ;>1 / 4$ a $1 / 2 ;>1 / 2$ a $1 ;>1$ a 2 ; $>2$ ); horas trabalhadas por semana em todos os trabalhos (até 14; 15 a 39; 40 a 44; 45 a 48; 49 ou mais); tempo de locomoção da residência até o trabalho (até 30 minutos; > 30 minutos a 1 hora; $>1$ hora a $2 ;>2$ horas); tempo no trabalho (até 1 ano; > 1 ano); "tinha carteira de trabalho assinada no trabalho?” (sim; não); contribuição previdenciária no trabalho? (sim; não); "realizava esforço físico intenso no trabalho?" (sim; não; não sabe); “cuidava dos afazeres domésticos?” (sim; não); número de horas dedicadas por semana aos afazeres domésticos.

A questão sobre esforço físico no trabalho incluiu pessoas a partir de 14 anos, sendo a pergunta: "no seu trabalho, anda a maior parte do tempo, carrega peso ou faz outra atividade que requer esforço físico intenso?". Os dados sobre as características de trabalho e rendimento consideraram o trabalho principal exercido na semana de referência de 21 a 27 de setembro de 2008, sendo de $\mathrm{R} \$ 415,00$ (quatrocentos e quinze reais) o valor do salário mínimo no mês de referência da pesquisa (setembro).

\section{Variáveis dependentes}

(a) Condições de saúde e morbidade referidas: estado de saúde autorreferido (muito bom/bom; regular/ruim/muito ruim); "deixou de realizar atividades habituais nas duas últimas semanas por motivo de saúde?" (sim; não); "esteve internado nos últimos 12 meses?” (sim; não).

(b)Doença crônica: doença de coluna ou costas; hipertensão; tendinite ou tenossinovite; depressão; artrite ou reumatismo. As doenças crônicas foram investigadas mediante a pergunta: "algum médico ou profissional de saúde disse que tinha alguma das seguintes doenças?”, tendo resposta "sim ou não".
A variável que corresponde ao informante das características de saúde e morbidade referidas apresentou a seguinte codificação $(0=$ própria pessoa, 1 = não morador; 2 = outro morador; 3 = ignorado).

\section{Análise dos dados}

Foi realizada análise das características do trabalho principal de crianças e adolescentes segundo faixa etária, utilizando distribuição proporcional e intervalo de 95\% de confiança (IC95\%). Também foi realizada análise comparativa das variáveis demográficas, socioeconômicas, saúde e morbidade referida entre as categorias de análise: "trabalhadores e não trabalhadores", mediante distribuição proporcional e IC95\%. Foram estimadas razões de prevalência brutas e ajustadas por sexo, idade, macrorregião de residência e informante das questões de saúde entre as categorias de análise que compõem a variável independente principal e as características de saúde por meio de regressão de Poisson, sendo os "não trabalhadores" a categoria de referência (IC95\% e nível de significância de 5\%).

A escolha das faixas etárias de 5 a 9 e 10 a 17 anos para análise das características do trabalho seguiu a própria metodologia da PNAD, uma vez que ela apresenta um módulo específico de perguntas sobre trabalho e rendimentos para o grupo de crianças de 5 a 9 anos e outro para pessoas a partir de 10 anos, quando se pesquisa a participação na população economicamente ativa (composta por pessoas ocupadas ou desocupadas) 21.

Por se tratar de amostra complexa, todas as análises foram realizadas no módulo survey do software estatístico Stata 9.2 (StataCorp., College Station, Estados Unidos), utilizando as ponderações necessárias. Cabe ressaltar que informações não declaradas foram consideradas missing, e seus valores não foram utilizados nas estimações realizadas.

O estudo obteve aprovação do Comitê de Ética em Pesquisa da Faculdade de Ciências Médicas da Universidade Estadual de Campinas (parecer no 252.058).

\section{Resultados}

\section{Características demográficas e socioeconômicas}

Das 91.377 crianças e adolescentes de 5 a 17 anos analisados do banco de dados da PNAD/2008, $11.002(12,34 \%)$ trabalhavam ou estavam em busca de emprego na semana de referência da pesquisa (IC95\%: 12,11-12,58). 
Comparados aos "não trabalhadores", os "trabalhadores" apresentaram maior proporção de meninos; faixa etária de 14 a 17 anos; pretos e pardos; residência na Região Nordeste; rendimentos domiciliares acima de $1 / 2$ a 2 salários mínimos e proporção 3,6 vezes maior de ausência de frequência escolar. Não há sobreposição dos intervalos de confiança das categorias de cada variável segundo condição de ocupação (Tabela 1).

Apesar do predomínio de melhores condições de moradia e saneamento, crianças e adolescentes que trabalhavam ou buscavam por emprego apresentaram maior proporção de domicílios sem água canalizada; sem banheiro; sem coleta adequada de lixo; com menor posse de bens essenciais, como geladeira, e com maior proporção de domicílios cadastrados no Programa Saúde da Família (PSF) quando comparados àqueles que não trabalhavam, sem sobreposição dos intervalos de confiança (Tabela 1).

\section{Características do trabalho principal (crianças de 5 a 9 anos)}

A maioria das crianças de 5 a 9 anos que possuíam trabalho residiam na zona rural e se encontravam majoritariamente em atividades agrícolas. Os segmentos de comércio e reparação e indústria de transformação foram os que mais concentraram crianças nos empreendimentos não agrícolas. O trabalho não remunerado em auxílio a membro da unidade domiciliar também foi predominante, e mais da metade auxiliava nos afazeres domésticos (Tabela 2), com cargas horárias que variaram de 1 a 42 horas semanais ( édia $=7,16$; mediana $=7$ ). O número de horas habitualmente trabalhadas na semana em todos os trabalhos variou de 1 hora a 44 horas (média = 12,05; mediana $=10$ ).

Características do trabalho principal (crianças e adolescentes de 10 a 17 anos)

A maioria das crianças e adolescentes de 10 a 17 anos ocupados vivia em zonas urbanas (Tabela 3). Estratificando por idade, verifica-se que $56,55 \%$ na faixa de 10 a 13 anos ( $n=995$; IC95\%: 54,07-59,01) residiam em zona rural, sendo esse percentual menor (30,37\%) entre os adolescentes de 14 a 17 anos ( $n=2.135$; IC95\%: 29,23-31,53).

Apesar de o trabalho exercido por eles ser majoritariamente em atividades não agrícolas (Tabela 3), verifica-se novamente distinção por idade: pessoas de 10 a 13 anos concentravamse em atividades agrícolas ( $\mathrm{n}=998 ; 58,47 \%$; IC95\%: 55,99-60,90), já as de 14 a 17 anos em atividades não agrícolas $(n=5.080 ; 71,71 \%$; IC95\%: 70,56-72,83).
Entre os trabalhadores agrícolas, os não remunerados e trabalhadores para o próprio consumo eram a maioria. Para aqueles que exerciam suas atividades em empreendimentos não agrí colas, a maioria era empregada, sendo o comércio e reparação e indústria de transformação os grupamentos de atividades em que mais se concentraram (Tabela 3).

Mais de $80 \%$ das crianças e adolescentes recebiam até 1 salário por seu trabalho, e mais da metade trabalhava de 15 a 44 horas. A maioria gastava até 30 minutos para chegar ao trabalho, sendo que $20 \%$ referiram um intervalo de deslocamento maior. Ainda que aproximadamente $40 \%$ delas estivessem há mais de um ano no trabalho atual, a maioria estava alheia às legislações trabalhistas e aos benefícios sociais, uma vez que a maioria não possuía carteira de trabalho assinada ou não contribuía para a previdência social. Além disso, 40\% dos adolescentes consideraram seu trabalho fisicamente pesado (Tabela 3). Mais da metade cuidava também dos afazeres domésticos (Tabela 3), dedicando de 1 a 40 horas semanais (média $=10,78$; mediana $=8$ ).

\section{Saúde e morbidade referidas}

Comparados aos que não trabalhavam, crianças e adolescentes que trabalhavam ou buscavam emprego tiveram prevalência maior de pior estado de saúde referido; internações nos 12 últimos meses e apresentaram maior relato de doença de coluna ou costas; hipertensão; tendinite ou tenossinovite; depressão e artrite ou reumatismo (Tabela 4).

$\mathrm{Na}$ análise das razões de prevalência ajustadas, estiveram associados ao trabalho infantil: pior estado de saúde referido; afastamento das atividades habituais por motivo de saúde; doença de coluna ou costas; depressão; atrite ou reumatismo (Tabela 5). O informante das características de saúde e morbidade foi, em sua maioria outra pessoa moradora do domicílio $(73,9 \%)$.

\section{Discussão}

Crianças e adolescentes em situação de trabalho ou em busca de emprego apresentaram piores condições de vida do que crianças não trabalhadoras, destacando-se a menor frequência escolar.

Estatísticas nacionais e internacionais também mostram maior proporção de meninos e adolescentes entre 14 e 17 anos trabalhando 15 . De um modo geral, meninos estão mais sujeitos a trabalhos pesados e perigosos, sendo mais vulneráveis a doenças e acidentes relacionados ao trabalho ${ }^{3}$. Entretanto, meninas também execu- 
Tabela 1

Distribuição proporcional das variáveis demográficas, socioeconômicas e características da unidade domiciliar entre crianças e adolescentes de 5 a 17 anos segundo condição de ocupação ( $\mathrm{n}=$ 91.377). Pesquisa Nacional por Amostra de Domicílios, Brasil, 2008 *.

\begin{tabular}{|c|c|c|c|c|c|c|}
\hline \multirow[t]{2}{*}{ Variáveis } & \multicolumn{3}{|c|}{ Não trabalhadores } & \multicolumn{3}{|c|}{ Trabalhadores } \\
\hline & $\mathbf{n}$ & $\%$ & IC95\% & $\mathbf{n}$ & $\%$ & IC95\% \\
\hline \multicolumn{7}{|l|}{ Características demográficas e socioeconômicas } \\
\hline \multicolumn{7}{|l|}{ Sexo } \\
\hline Feminino & 40.556 & 50,55 & $50,17-50,93$ & 4.063 & 37,30 & $36,32-38,30$ \\
\hline Masculino & 39.819 & 49,45 & $49,07-49,83$ & 6.939 & 62,70 & $61,70-63,68$ \\
\hline \multicolumn{7}{|l|}{ Faixa etária (anos) } \\
\hline $5-9$ & 32.839 & 40,87 & $40,50-41,25$ & 291 & 2,62 & $2,32-2,96$ \\
\hline $10-13$ & 27.374 & 34,22 & $33,86-34,58$ & 1.924 & 17,08 & $16,35-17,85$ \\
\hline $14-17$ & 20.162 & 24,91 & $24,58-25,24$ & 8.787 & 80,29 & $79,48-81,08$ \\
\hline \multicolumn{7}{|l|}{ Cor ou raça autorreferida ** } \\
\hline Branca & 32.875 & 44,22 & $43,83-44,60$ & 3.964 & 39,06 & $38,06-40,07$ \\
\hline Pretos e pardos & 46.972 & 55,12 & $54,74-55,51$ & 6.900 & 60,20 & $59,18-61,2$ \\
\hline Amarela & 299 & 0,43 & $0,38-0,49$ & 29 & 0,26 & $0,17-0,39$ \\
\hline Indígena & 201 & 0,22 & $0,19-0,26$ & 54 & 0,47 & $0,35-0,63$ \\
\hline \multicolumn{7}{|l|}{ Macrorregião de residência } \\
\hline Sudeste & 21.146 & 38,49 & $38,10-38,88$ & 2.518 & 32,53 & $31,51-33,56$ \\
\hline Sul & 10.627 & 13,26 & $13,01-13,51$ & 1.603 & 15,59 & $14,87-16,34$ \\
\hline Centro-oeste & 8.859 & 7,36 & $7,21-7,51$ & 1.246 & 7,74 & $7,32-8,18$ \\
\hline Norte & 12.739 & 10,09 & $9,90-10,28$ & 1.676 & 9,35 & $8,87-9,86$ \\
\hline Nordeste & 27.004 & 30,80 & $30,46-31,14$ & 3.959 & 34,78 & $33,84-35,74$ \\
\hline \multicolumn{7}{|c|}{ Faixa de rendimento mensal domiciliar per capita } \\
\hline \multicolumn{7}{|l|}{ (salários mínimos) ${ }^{\star \star \star}$} \\
\hline$>2$ & 7.207 & 9,29 & $9,06-9,52$ & 590 & 6,09 & $5,59-6,64$ \\
\hline$>1$ a 2 & 11.876 & 16,26 & $15,97-16,56$ & 1.834 & 18,55 & $17,73-19,39$ \\
\hline$>1 / 2$ a 1 & 22.146 & 28,62 & $28,27-28,97$ & 3.305 & 30,80 & $29,85-31,77$ \\
\hline$>1 / 4$ a $1 / 2$ & 21.883 & 27,1 & $26,76-27,44$ & 2.776 & 25,31 & $24,44-26,21$ \\
\hline Até $1 / 4$ & 14.516 & 17,77 & $17,49-18,06$ & 2.083 & 18,71 & $17,94-19,50$ \\
\hline Sem rendimentos & 758 & 0,95 & 0,87-1,02 & 56 & 0,53 & 0,39-0,71 \\
\hline \multicolumn{7}{|l|}{ Frequenta escola ou creche? } \\
\hline Sim & 76.096 & 94,97 & $94,08-95,13$ & 8.968 & 81,51 & $80,70-82,29$ \\
\hline Não & 4.279 & 5,03 & $4,87-5,19$ & 2.034 & 18,49 & $17,71-19,30$ \\
\hline \multicolumn{7}{|l|}{ Características da unidade domiciliar } \\
\hline \multicolumn{7}{|l|}{ Domicílio possui água canalizada? \# } \\
\hline Sim & 71.940 & 89,81 & $89,59-90,04$ & 8.915 & 81,95 & $81,17-82,70$ \\
\hline Não & 8.255 & 10,19 & $9,96-10,41$ & 2.057 & 18,05 & $17,30-18,83$ \\
\hline \multicolumn{7}{|c|}{ Presença de banheiro ou sanitário no domicílio? \# } \\
\hline $\operatorname{Sim}$ & 76.006 & 94,60 & $94,42-94,77$ & 9.833 & 89,62 & $88,99-90,22$ \\
\hline Não & 4.189 & 5,40 & $5,23-5,57$ & 1.139 & 10,38 & $9,78-11,01$ \\
\hline \multicolumn{7}{|l|}{ Destino do lixo domiciliar\# } \\
\hline Coletado diretamente ou indiretamente & 68.747 & 85,41 & $85,14-85,67$ & 7.813 & 71,52 & $70,60-72,41$ \\
\hline Outro & 11.448 & 14,59 & $14,33-14,86$ & 3.159 & 28,48 & $27,59-29,40$ \\
\hline \multicolumn{7}{|l|}{ Possui geladeira? \# } \\
\hline Sim & 72.381 & 90,56 & $90,34-90,77$ & 9.119 & 84,13 & $83,40-84,94$ \\
\hline Não & 7.814 & 9,43 & $9,22-9,65$ & 1.853 & 15,87 & $15,16-16,60$ \\
\hline \multicolumn{7}{|l|}{ Domicílio cadastrado no programa de saúde } \\
\hline \multicolumn{7}{|l|}{ da família? \# } \\
\hline $\operatorname{Sim}$ & 43.944 & 54,87 & $54,49-55,26$ & 6.800 & 62,32 & $61,32-63,31$ \\
\hline Não & 36.251 & 45,13 & $44,74-45,51$ & 4.172 & 37,68 & $36,69-38,68$ \\
\hline
\end{tabular}

IC95\%: intervalo de 95\% de confiança.

* Valor de $p<0,01$ no teste qui-quadrado de Pearson;

** Sem declaração $(n=83)$;

*** Sem declaração e missing $(n=2.347)$;

\# Não aplicável $(n=210)$. 
Distribuição proporcional das crianças de 5 a 9 anos trabalhadoras, segundo características do trabalho principal da semana de referência da pesquisa $(n=291)$. Pesquisa Nacional por Amostra de Domicílios, Brasil, 2008.

\begin{tabular}{|c|c|c|c|}
\hline Variáveis & $\mathbf{n}$ & $\%$ & IC95\% \\
\hline \multicolumn{4}{|l|}{ Situação de residência } \\
\hline Urbana & 87 & 28,78 & $23,54-34,65$ \\
\hline Rural & 204 & 71,22 & $65,35-76,46$ \\
\hline \multicolumn{4}{|l|}{ Atividade principal do empreendimento } \\
\hline Não agrícola & 91 & 26,80 & $21,84-32,43$ \\
\hline Agrícola & 200 & 73,20 & $67,57-78,16$ \\
\hline \multicolumn{4}{|l|}{ Grupamentos de atividade principal do empreendimento } \\
\hline Agricultura e pecuária & 200 & 73,20 & $67,57-78,16$ \\
\hline Indústria de transformação & 29 & 7,25 & $4,92-10,54$ \\
\hline Construção & 4 & 1,03 & $0,34-3,02$ \\
\hline Comércio e reparação & 38 & 11,81 & $8,44-16,28$ \\
\hline Alojamento, alimentação & 10 & 3,58 & $1,82-6,90$ \\
\hline Outros & 10 & 3,15 & $1,55-6,24$ \\
\hline \multicolumn{4}{|l|}{ Posição na ocupação no trabalho principal } \\
\hline Empregado & 9 & 3,86 & $1,92-7,59$ \\
\hline Trabalhador doméstico & 3 & 0,78 & $0,21-2,80$ \\
\hline Conta própria & 10 & 3,71 & $1,91-7,10$ \\
\hline Não remunerado membro da unidade domiciliar & 208 & 71,49 & $65,56-76,76$ \\
\hline Outro trabalhador não remunerado & 8 & 2,75 & $1,35-5,50$ \\
\hline $\begin{array}{l}\text { Trabalhador na produção para próprio consumo ou na } \\
\text { construção para próprio uso }\end{array}$ & 53 & 17,40 & $13,23-22,55$ \\
\hline \multicolumn{4}{|l|}{ Cuidava dos afazeres domésticos? } \\
\hline Não & 118 & 39,34 & $33,52-45,49$ \\
\hline Sim & 173 & 60,66 & $54,51-66,48$ \\
\hline
\end{tabular}

IC95\%: intervalo de 95\% de confiança.

tam tarefas de elevado risco de acidente e adoecimento, sendo as mais expostas ao trabalho infantil doméstico e à exploração sexual 22.

A maior proporção de crianças e adolescentes negros e pardos entre aqueles em situação laboral é o reflexo das desigualdades raciais ainda presentes 23 . Eles também apresentam maior vulnerabilidade aos diversos tipos de desigualdades quando comparados às crianças brancas, como menor frequência escolar 24 .

A Região Nordeste foi a macrorregião de maior proporção entre as crianças que trabalhavam ou estavam em busca de emprego, como já referido em análises nacionais 9 . Menor distribuição de renda, maior concentração de pobreza e questões culturais podem ser responsáveis por essa estatística na região, além do descaso das autoridades estatais e ausência de políticas públicas de apoio às famílias mais vulneráveis 25 .

Famílias com crianças e adolescentes que trabalhavam ou buscavam por emprego tiveram menor proporção de rendimentos per capita superiores a dois salários mínimos e maior proporção na faixa da pobreza e indigência. Análise das PNADs (1992 a 2003) identificou influência do aumento na renda domiciliar na diminuição do trabalho de crianças de 5 a 9 anos, entretanto, na faixa etária de 10 a 17 anos, essa redução só foi vista com rendimentos acima de $\mathrm{R} \$ 1.800,0023$. Apesar da influência das desigualdades econômicas sobre o trabalho infantil, a pobreza, medida pela renda familiar, não pode ser considerada o único fator para sua ocorrência nem o único foco de políticas sociais de combate ao trabalho infantil 26.

A redução na frequência escolar é um efeito conhecido do trabalho infantil que pode gerar menor renda na fase adulta, pois reduz o nível de escolarização e, consequentemente, a qualificação profissional 11,27,28. Neste estudo, a menor proporção de frequência escolar entre crianças e adolescentes trabalhadores comparados aos 
Tabela 3

Distribuição proporcional das crianças e adolescentes de 10 a 17 anos ocupados, segundo características do trabalho principal da semana de referência da pesquisa $(n=8.813)$. Pesquisa Nacional por Amostra de Domicílios, Brasil, 2008.

\begin{tabular}{|c|c|c|c|}
\hline Variáveis & $\mathrm{n}$ & $\%$ & $\mathrm{IC95 \%}$ \\
\hline \multicolumn{4}{|l|}{ Situação de residência } \\
\hline Urbana & 5.683 & 64,46 & $63,38-65,53$ \\
\hline Rural & 3.130 & 35,54 & $34,47-36,62$ \\
\hline \multicolumn{4}{|l|}{ Atividade principal do empreendimento } \\
\hline Não agrícola & 5.864 & 65,75 & $64,67-66,81$ \\
\hline Agrícola & 2.949 & 34,25 & $33,19-35,33$ \\
\hline \multicolumn{4}{|l|}{ Posição na ocupação (trabalhador agrícola) } \\
\hline Empregador nos serviços auxiliares & 1 & 0,03 & $0,003-0,18$ \\
\hline Empregado permanente & 167 & 5,72 & $4,89-6,68$ \\
\hline Empregado temporário & 280 & 10,10 & $8,99-11,33$ \\
\hline Conta própria & 63 & 2,09 & $1,60-2,72$ \\
\hline Não remunerado membro da unidade domiciliar e outro (não remunerado) & 1.649 & 56,60 & $54,70-58,48$ \\
\hline Trabalhador na produção para o próprio consumo & 789 & 25,46 & $23,83-27,15$ \\
\hline \multicolumn{4}{|l|}{ Posição na ocupação (trabalhador não agrícola) } \\
\hline Empregado & 3.464 & 61,12 & $59,75-62,47$ \\
\hline Trabalhador doméstico & 695 & 11,42 & $10,58-12,32$ \\
\hline Conta própria & 576 & 9,20 & $8,45-10,02$ \\
\hline Empregador & 7 & 0,09 & $0,04-0,21$ \\
\hline Não remunerado membro da unidade domiciliar e outro (não remunerado) & 1.086 & 17,53 & $16,51-18,61$ \\
\hline Trabalhador na construção para o próprio uso & 36 & 0,62 & $0,43-0,89$ \\
\hline \multicolumn{4}{|l|}{ Grupamentos de atividade principal } \\
\hline Educação, saúde e serviços sociais & 240 & 2,72 & $2,36-3,11$ \\
\hline Agrícola & 2.949 & 34,25 & $33,19-35,33$ \\
\hline Outras atividades industriais & 33 & 0,35 & $0,23-0,51$ \\
\hline Indústria de transformação & 907 & 10,23 & $9,56-10,94$ \\
\hline Construção & 467 & 5,42 & $4,92-5,96$ \\
\hline Comércio e reparação & 1.925 & 21,54 & $20,63-22,49$ \\
\hline Alojamento, alimentação & 514 & 5,87 & $5,34-6,43$ \\
\hline Transporte, armazenagem e comunicação & 192 & 2,25 & $1,92-2,61$ \\
\hline Administração pública & 108 & 1,05 & $0,85-1,30$ \\
\hline Serviços domésticos & 695 & 7,51 & $6,94-8,11$ \\
\hline Outros serviços coletivos, sociais ou pessoais & 346 & 3,90 & $3,48-4,36$ \\
\hline Outras atividades & 384 & 4,47 & $4,01-4,98$ \\
\hline Atividades mal definidas & 53 & 0,44 & $0,32-0,59$ \\
\hline \multicolumn{4}{|l|}{ Rendimento mensal de todos os trabalhos em salários mínimos * } \\
\hline$>2$ & 46 & 0,48 & $0,35-0,66$ \\
\hline$>1$ a 2 & 711 & 9,20 & $8,52-9,92$ \\
\hline$>1 / 2$ a 1 & 1.790 & 20,61 & $19,70-21,56$ \\
\hline$>1 / 4$ a $1 / 2$ & 1.299 & 14,32 & $13,55-15,13$ \\
\hline Sem rendimentos a $1 / 4$ & 4.923 & 55,38 & $54,24-56,51$ \\
\hline \multicolumn{4}{|l|}{ Horas habitualmente trabalhadas por semana } \\
\hline Até 14 & 1.975 & 21,54 & $20,64-22,46$ \\
\hline 15 a 39 & 4.196 & 47,39 & $46,26-48,52$ \\
\hline 40 a 44 & 1.503 & 17,75 & $16,89-18,65$ \\
\hline 45 a 48 & 557 & 6,64 & $6,08-7,24$ \\
\hline 49 ou mais & 582 & 6,68 & $6,13-7,28$ \\
\hline
\end{tabular}

(continua) 
Tabela 3 (continuação)

\begin{tabular}{|c|c|c|c|}
\hline Variáveis & n & $\%$ & IC95\% \\
\hline \multicolumn{4}{|c|}{ Tempo de locomoção da residência até o local de trabalho ** } \\
\hline Até 30 minutos & 4.188 & 79,76 & $78,53-80,94$ \\
\hline$>30$ minutos a 1 hora & 729 & 14,16 & $13,15-15,23$ \\
\hline$>1$ hora a 2 horas & 256 & 5,06 & $4,43-5,79$ \\
\hline$>2$ horas & 52 & 1,00 & $0,74-1,36$ \\
\hline \multicolumn{4}{|c|}{ Tempo no trabalho principal } \\
\hline Até um ano & 5.417 & 61,56 & $60,46-62,65$ \\
\hline Mais de 1 ano & 3.396 & 38,44 & $37,35-39,54$ \\
\hline \multicolumn{4}{|c|}{ Tinha carteira de trabalho assinada? } \\
\hline Sim & 370 & 8,98 & $8,08-9,97$ \\
\hline Não & 4.236 & 91,01 & $90,02-91,91$ \\
\hline \multicolumn{4}{|c|}{ Contribuição previdenciária no trabalho } \\
\hline Sim & 718 & 9,14 & $8,47-9,86$ \\
\hline Não & 8.095 & 90,86 & $90,14-91,53$ \\
\hline \multicolumn{4}{|c|}{ Esforço físico intenso no trabalho *** } \\
\hline Sim & 2.676 & 38,46 & $37,23-39,70$ \\
\hline Não & 4.281 & 60,69 & $59,45-61,93$ \\
\hline Não sabe & 60 & 0,94 & $0,64-1,10$ \\
\hline \multicolumn{4}{|c|}{ Cuidava dos afazeres domésticos? } \\
\hline Não & 3.724 & 42,97 & $41,85-44,09$ \\
\hline Sim & 5.089 & 57,03 & $55,91-58,15$ \\
\hline
\end{tabular}

* Missing $(n=44)$;

** Questionado aos 5.225 que não moravam em domicílio que estava no mesmo terreno ou área do estabelecimento que trabalhava. Não inclui trabalhadores na produção para o próprio consumo e uso;

*** Das 7.031 crianças maiores de 14 anos ocupadas, houve resposta para 7.017 delas.

não trabalhadores apontam para desigualdades socioeconômicas no trabalho infantil. Analisando dados do suplemento da PNAD/2006, Carvalho 28 identificou que a frequência escolar de pessoas de 5 a 17 anos ocupadas era inferior à registrada para a população na mesma faixa etária em áreas urbanas e rurais, além de maior atraso escolar. Estudo incluindo adolescentes encontrou associação entre trabalho infantil e repetição de, pelo menos, uma série escolar 29.

A maior parte dos domicílios com crianças e adolescentes trabalhadores possuía cadastro no PSF do Governo Federal. A proximidade com as famílias e a facilidade em realizar o diagnóstico situacional das áreas de abrangência são tarefas das equipes que compõem esse programa. Cabe a elas investigar a ocorrência desse evento e, juntamente com programas de prevenção e erradicação do trabalho infantil, auxiliar crianças, adolescentes e suas famílias, investigando problemas de saúde que possam estar associados ao trabalho infantil.

Analisando de uma forma geral as características do trabalho das crianças de 5 a 9 anos, as atividades no setor agrícola são as que mais utilizam esse grupo etário, principalmente co- mo trabalhadores não remunerados membros das unidades domiciliares, que, por definição do IBGE, são aqueles que executam tarefas de, ao menos, 1 hora por semana em ajuda a outros membros da família 21,30.

Kassouf \& Santos 31 identificaram aumento da probabilidade de crianças trabalharem em atividades agrícolas e pecuárias com o incremento do tamanho da propriedade agrícola até atingir um ponto de 76 hectares, ressaltando a necessidade de se aprofundar a investigação da relação entre riqueza familiar e trabalho infantil agrícola.

Fatores culturais influenciam para que o trabalho infantil rural seja visto de forma naturalizada ou desejada pelos membros da família, existindo aceitação generalizada de que seria parte da formação profissional das crianças desde a tenra idade. Entretanto, esse fato deve ser observado com cautela, pois, além de algumas condições de trabalho no meio rural serem perigosas $\mathrm{e}$ proibidas para menores de 18 anos, como a manipulação de agrotóxicos, não é raro encontrar casos de exploração sexual, violência física e até trabalho em cultivo ilícito 32 . 
Prevalência das características de saúde e morbidade referida entre crianças e adolescentes de 5 a 17 anos ( $n=91.377)$. Pesquisa Nacional por Amostra de Domicílios, Brasil, 2008

\begin{tabular}{|c|c|c|c|c|c|c|c|}
\hline \multirow[t]{2}{*}{ Variáveis } & \multicolumn{3}{|c|}{ Não trabalhadores } & \multicolumn{3}{|c|}{ Trabalhadores } & \multirow[t]{2}{*}{ Valor de p * } \\
\hline & $\mathbf{n}$ & $\%$ & IC95\% & $\mathbf{n}$ & $\%$ & IC95\% & \\
\hline \multicolumn{8}{|l|}{ Estado de saúde autorreferido } \\
\hline Muito bom/Bom & 73.261 & 91,47 & $91,26-91,68$ & 9.794 & 89,58 & $88,96-90,17$ & $<0,01$ \\
\hline Regular/Ruim e Muito ruim & 7.114 & 8,52 & $8,32-8,74$ & 1.208 & 10,42 & $9,82-11,04$ & \\
\hline \multicolumn{8}{|c|}{$\begin{array}{l}\text { Afastou das atividades habituais por motivo de saúde nas } \\
\text { duas últimas semanas? }\end{array}$} \\
\hline Não & 75.282 & 93,69 & $93,5-93,87$ & 10.338 & 94,03 & $93,53-94,49$ & 0,20 \\
\hline Sim & 5.093 & 6,31 & $6,12-6,49$ & 664 & 5,96 & $5,50-6,46$ & \\
\hline \multicolumn{8}{|c|}{ Esteve internado nos últimos 12 meses? } \\
\hline Não & 77.391 & 96,33 & $96,19-96,47$ & 10.527 & 95,69 & $95,26-96,08$ & $<0,01$ \\
\hline Sim & 2.984 & 3,66 & $3,52-3,81$ & 475 & 4,31 & $3,92-4,74$ & \\
\hline \multicolumn{8}{|l|}{ Doença crônica referida } \\
\hline \multicolumn{8}{|l|}{ Doença de coluna ou costas } \\
\hline Não & 79.313 & 98,71 & $98,62-98,79$ & 10.685 & 97,05 & $96,68-97,38$ & $<0,01$ \\
\hline Sim & 1.062 & 1,29 & $1,21-1,38$ & 317 & 2,94 & $2,61-3,13$ & \\
\hline \multicolumn{8}{|l|}{ Hipertensão } \\
\hline Não & 80.157 & 99,74 & $99,69-99,77$ & 10.930 & 99,34 & $99,15-99,49$ & $<0,01$ \\
\hline Sim & 218 & 0,26 & $0,22-0,30$ & 72 & 0,66 & $0,51-0,84$ & \\
\hline \multicolumn{8}{|l|}{ Tendinite ou tenossinovite } \\
\hline Não & 80.255 & 99,84 & $99,80-99,87$ & 10.966 & 99,58 & $99,41-99,71$ & $<0,01$ \\
\hline Sim & 120 & 0,16 & $0,13-0,19$ & 36 & 0,41 & $0,29-0,59$ & \\
\hline \multicolumn{8}{|l|}{ Depressão } \\
\hline Não & 80.084 & 99,64 & $99,59-99,68$ & 10.912 & 99,14 & $98,93-99,32$ & $<0,01$ \\
\hline Sim & 291 & 0,36 & $0,31-0,41$ & 90 & 0,85 & $0,68-1,07$ & \\
\hline \multicolumn{8}{|l|}{ Artrite ou reumatismo } \\
\hline Não & 80.049 & 99,62 & $99,57-99,66$ & 10.901 & 99,14 & $98,94-99,31$ & $<0,01$ \\
\hline Sim & 326 & 0,38 & $0,33-0,43$ & 101 & 0,85 & $0,69-1,05$ & \\
\hline
\end{tabular}

IC95\%: intervalo de 95\% de confiança.

* Teste qui-quadrado de Pearson.

O predomínio de cargas horárias superiores a uma hora por semana em todos os trabalhos e nos afazeres domésticos aponta para a dupla jornada de trabalho dos menores de 10 anos, levando a crer que suas atividades vão além do simples auxílio aos demais trabalhadores, contribuindo possivelmente para a subsistência das famílias 23 .

Conforme identificado em séries históricas, com aumento da idade, há uma redução da ocupação das crianças e adolescentes no trabalho em áreas agrícolas e aumento nos setores urbanos 33. Não diferente, encontramos maior proporção de adolescentes a partir de 14 anos de idade em atividades não agrícolas.

Entre os ocupados de 10 a 17 anos em atividades não agrícolas, a condição de "empregado" foi a mais frequente, sendo os setores de comércio e reparação e indústria de transformação os de maior proporção. Em análise de suplemento especial sobre trabalho infantil da PNAD/2001, Kassouf 34 mostrou que esses setores estão entre os de maior proporção de lesões e acidentes entre crianças e adolescentes, sendo cortes, dores musculares e fraturas as principais ocorrências relatadas nos setores industriais e comércio.

As remunerações eram muito baixas, sendo que a carga horária em sua maioria era acima de 14 horas semanais. É de se supor que o valor pago pelas atividades não seja condizente com o tempo ou com as atividades realizadas, uma vez que crianças são, conhecidamente, mão de obra fácil, barata e dócil, sendo facilmente reprimidas e tolhidas a aceitar o trabalho e a remuneração ${ }^{15}$.

O tempo de deslocamento até o trabalho é uma característica adicional das condições de trabalho e qualidade de vida do trabalhador 11 
Tabela 5

Razões de prevalência das características de saúde e morbidade referida entre crianças e adolescentes de 5 a 17 anos trabalhadores ( $\mathrm{n}=91.377$ ). Pesquisa Nacional por Amostra de Domicílios, Brasil, 2008.

\begin{tabular}{|c|c|c|c|c|}
\hline Variáveis & RP bruta & IC95\% & RP ajustada * & IC95\% \\
\hline \multicolumn{5}{|l|}{ Estado de saúde autorreferido } \\
\hline Muito bom/Bom & 1,00 & & 1,00 & \\
\hline Regular/Ruim e Muito ruim & 1,24 & $1,17-1,31$ & 1,18 & $1,11-1,26$ \\
\hline \multirow{2}{*}{\multicolumn{5}{|c|}{$\begin{array}{l}\text { Afastou das atividades habituais por motivo de saúde nas duas } \\
\text { últimas semanas? }\end{array}$}} \\
\hline & & & & \\
\hline Não & 1,00 & & 1,00 & \\
\hline Sim & 0,95 & $0,88-1,03$ & 1,17 & $1,07-1,27$ \\
\hline \multicolumn{5}{|c|}{ Esteve internado nos últimos 12 meses? } \\
\hline Não & 1,00 & & 1,00 & \\
\hline Sim & 1,16 & $1,05-1,27$ & 1,10 & $0,99-1,22$ \\
\hline \multicolumn{5}{|l|}{ Doença crônica referida } \\
\hline \multicolumn{5}{|l|}{ Doença de coluna ou costas } \\
\hline Não & 1,00 & & 1,00 & \\
\hline Sim & 2,18 & $1,92-2,46$ & 1,19 & $1,04-1,36$ \\
\hline \multicolumn{5}{|l|}{ Hipertensão } \\
\hline Não & 1,00 & & 1,00 & \\
\hline Sim & 2,41 & $1,84-3,14$ & 1,36 & $0,98-1,88$ \\
\hline \multicolumn{5}{|l|}{ Tendinite ou tenossinovite } \\
\hline Não & 1,00 & & 1,00 & \\
\hline Sim & 2,19 & $1,51-3,17$ & 1,14 & $0,76-1,72$ \\
\hline \multicolumn{5}{|l|}{ Depressão } \\
\hline Não & 1,00 & & 1,00 & \\
\hline Sim & 2,25 & $1,78-2,85$ & 1,37 & $1,06-1,77$ \\
\hline \multicolumn{5}{|l|}{ Artrite ou reumatismo } \\
\hline Não & 1,00 & & 1,00 & \\
\hline Sim & 2,26 & $1,81-2,82$ & 1,46 & $1,14-1,87$ \\
\hline
\end{tabular}

IC95\%: intervalo de 95\% de confiança; RP: razão de prevalência.

* Ajustada por sexo, idade, região de residência e informante.

Nota: crianças não trabalhadoras foram categoria de referência.

sendo também relevante para analisar outro aspecto prejudicial do trabalho infantil. Apesar de ter sido encontrada maior proporção de crianças e adolescentes com deslocamentos inferiores a 30 minutos (indicando possivelmente que a maioria residia próxima ao local de trabalho), deve-se considerar que a variável se refere apenas ao tempo de ida, e que tempos superiores a 1 hora foram encontrados. Longos períodos de locomoção geram mais cansaço, reduzem o tempo de lazer e de convivência familiar 11, além de prejudicarem a frequência escolar.

A baixa proporção de carteira de trabalho assinada e de não contribuição previdenciária na maioria dos trabalhos mostra a falta de vínculos regulamentados, a ausência de direitos sociais e o aspecto de invisibilidade do trabalho infan- til, caracterizando uma situação de abandono e exploração alimentada pela precariedade do trabalho e a absorção das crianças no universo do trabalho informal em que se encontram famílias brasileiras alheias à cobertura de direitos trabalhistas e previdenciários 35 .

O tempo de permanência no trabalho é uma variável que reflete a estabilidade dos vínculos empregatícios 11. Entretanto, no que se refere ao trabalho de crianças e adolescentes, a ampliação desse tempo pode sugerir dificuldades em se sair da condição de trabalho. Neste estudo, quase $40 \%$ de crianças e adolescentes estavam ocupadas há mais de um ano na atividade principal, o que pode indicar a dependência da família em mantê-los em situação de trabalho ${ }^{15}$. A análise dessa variável ao longo do tempo pode ser útil 
para acompanhar os resultados de intervenções e políticas públicas sobre o trabalho infantil no âmbito geral e nas áreas urbanas e rurais, uma vez que relações de trabalho na zona rural podem ser mais duradouras 11 .

Os maiores de 10 anos também auxiliavam nos afazeres domésticos, mostrando uma dupla carga de trabalho 30 que pode comprometer a frequência e o rendimento escolar. Aproximadamente, $8 \%$ dos que tinham idade acima de 10 anos eram ocupados em serviços domésticos. Trata-se de um fenômeno de difícil combate, uma vez que ocorre às portas fechadas no interior de muitos domicílios que empregam esse tipo de trabalho até mesmo sob o consentimento da sociedade 36 . Segundo a OIT, duas em cada três crianças que trabalham no serviço doméstico em casa de terceiros estão abaixo da idade legal ou expostas a situações de perigo. Em muitos casos, ficam longe de suas famílias e comunidades, chegando a sofrer maus-tratos e sendo vítimas de tráfico de seres humanos 22 .

O trabalho de adolescentes a partir de 14 anos foi considerado pesado em aproximadamente $40 \%$ dos casos. No geral, seja em áreas urbanas ou rurais, doméstico ou não, o trabalho infantil pode apresentar uma face desgastante e ainda mais danosa: são inúmeros os relatos de crianças que passam o dia andando, carregando peso acima de suas capacidades físicas, operando maquinário perigoso e pesado 27.

Apesar da dificuldade em se identificar pelo banco de dados o envolvimento de crianças e adolescentes nas piores formas de trabalho infantil, ou seja, aquelas definidas na Convenção $n^{\circ}$ 182 da OIT como atividades que expõem a abuso físico, emocional, sexual ou a riscos no ambiente de trabalho 37, é possível dizer que, pela reduzida idade, elevada carga horária de trabalho e envolvimento em atividades nos setores agrícolas, indústria de transformação, comércio e reparação, essas crianças sejam vítimas da exploração do trabalho infantil, ficando extremamente vulneráveis a adoecimentos e acidentes 28 .

Houve associação entre crianças e adolescentes em situação de trabalho ou em busca por emprego com pior saúde referida; restrição das atividades por motivo de saúde, depressão e doenças relacionadas ao aparelho musculoesquelético. Obter informações de crianças e familiares sobre trabalho infantil e conhecer seus efeitos sobre a saúde é um desafio, mas algumas evidências já apontam que tais impactos podem ser mais prejudiciais quanto menor a idade da criança, maior interferência em atividades escolares, maior jornada de trabalho e maior a exposição a riscos ocupacionais 38 . Estudos nacionais encontraram a associação entre trabalho infantil e dores musculoesqueléticas 39 ; problemas comportamentais (que poderiam envolver agressividade, ansiedade e depressão) 40 e maior chance de sofrer agressão física e ferimentos; sentir-se só 41 e ter problemas de sono 41,42 .

Diante dos dados, algumas considerações sobre as limitações do estudo devem ser feitas. Uma delas é a possível subestimação da população trabalhadora, uma vez que a pesquisa não contempla a população de rua, que, nesse caso, possui grande parte de crianças envolvidas nas piores formas de trabalho infantil 37. É factível considerar que, em alguns casos, os informantes das questões de trabalho sejam adultos que usufruam do trabalho infantil, não relatando o caso.

Por se tratar de um estudo transversal, a relação temporal entre as doenças e a situação de trabalho fica prejudicada, no entanto, as associações encontradas podem ser vistas como marcadores, sugerindo, aos profissionais de saúde, uma investigação do trabalho infantil, ou vulnerabilidade a ele, diante de crianças ou adolescentes com essas condições de saúde.

O uso do informante sobre as questões de saúde também merece discussão, uma vez que mais de $70 \%$ das respostas não foram fornecidas pelos próprios sujeitos da pesquisa. Porém, a inclusão da variável "tipo de informante" no modelo de regressão permitiu a redução desse viés no estudo. O uso do informante tem a vantagem de aumentar o universo amostral 43 , existindo concordância das respostas fornecidas por eles e as questões objetivas de saúde 44 .

\section{Conclusão}

Além das longas e duplas jornadas de trabalho em atividades que podem prejudicar sua saúde e segurança, crianças e adolescentes que trabalhavam ou estavam em busca de emprego estiveram expostos, de forma desigual, a piores situações socioeconômicas e a menor frequência escolar, apresentando pior saúde autorreferida e morbidades quando comprados aos que não trabalhavam.

O trabalho infantil representa ponto de estagnação ao desenvolvimento social e humano, refletindo uma estrutura de desigualdades evidenciada nas piores condições de vida das famílias. Tal estrutura é construída a partir de uma lógica perversa em que medidas macroeconômicas privilegiam a flexibilização dos processos de trabalho, a redução dos custos de produção, com consequente aumento de subempregos e trabalho infantil.

Esse fato aponta para uma intervenção imediata e cautelosa focada não apenas na eli- 
minação de postos de trabalho perigosos que empregam crianças e adolescentes, mas no fortalecimento da estrutura das famílias e acompanhamento com políticas e ações de apoio ao trabalho em zonas rurais e urbanas por equipes multiprofissionais de saúde e apoio intersetorial.

Cabe ressaltar que a PNAD/2008 não incluiu questões sobre programas de transferência de renda, e, apesar de estudos sugerirem que tais programas possuam impactos positivos na frequência escolar 45, estudos sobre esse efeito, em longo prazo, bem como a melhoria da qualidade de vida e saúde dessa população infantil são necessários.

\section{Resumen}

Los objetivos del estudio fueron describir el trabajo de niños y adolescentes brasileños y analizar la situación socioeconómica y la salud de los niños que trabajan y que no trabajan. De la Encuesta Nacional por Muestra de Domicilios (PNAD/2008) seleccionamos niños y adolescentes de 5 a 17 años y los dividimos en dos categorías de análisis: "trabajadores" (trabajando o buscando trabajo) y "no-trabajadores". Se calculó la prevalencia de las características del trabajo, así como las variables socioeconómicas y de salud en las categorías de análisis. Las razones de prevalencia ajustadas de las características de salud se estimaron mediante regresión de Poisson, los 'no trabajadores eran categoría de referencia. En comparación con los "no trabajadores", los "trabajadores" tenían mayor proporción de niños; 14 a 17 años; no caucásica; menor asistencia escolar y peores condiciones de vivienda. El trabajo infantil se asoció con una percepción de salud peor; enfermedades de columna o espalda; artritis o reumatismo y depresión. Las políticas eficaces de apoyo a las familias deben reforzarse para combatir eficazmente el trabajo infantil.

Encuestas Epidemiológicos; Desigualdades en la Salud; Trabajo de Menores

\section{Colaboradores}

I. O. C. Miquilin participou da concepção do estudo, análise e interpretação dos dados e redação do artigo. L. Marín-León participou da análise e interpretação dos dados e redação do artigo. V. G. Luz participou da análise e interpretação dos dados e redação do artigo. E. I. G. La-Rotta participou da análise e interpretação dos dados e redação do artigo. H. R. Corrêa Filho participou da concepção, análise e interpretação dos dados e redação do artigo.

\section{Agradecimentos}

À Coordenação de Aperfeiçoamento de Pessoal de Nível Superior (Capes) pela bolsa concedida à primeira autora. 


\section{Referências}

1. Dias EC, Oliveira RP, Machado JH, Minayo-Gomez C, Perez MAG, Hoefel MGL, et al. Employment conditions and health inequities: a case study of Brazil. Cad Saúde Pública 2011; 27:2452-60.

2. United Nations Children's Fund; International Labour Organization. Supporting workers with families responsabilites: conecting child development and the decent work agenda. New York: United Nations Children's Fund/International Labour Organization; 2012.

3. International Labour Organization. Marking progress against child labour: Global estimates and trends 2000-2012. Geneva: International Labour Organization; 2013.

4. Dias JC, Araújo GS. O trabalho infantil doméstico no Brasil: avaliação a partir dos microdados da Pnad/IBGE (2008-2011). Brasília: Fórum Nacional de Prevenção e Erradicação do Trabalho Infantil; 2013.

5. Instituto Brasileiro de Geografia e Estatística. Censo 2000 e 2010: trabalho infantil. http: / / censo2010. ibge.gov.br/trabalhoinfantil/outros/graficos.html (acessado em 22/Jul/2013).

6. Colucci V. A teoria da proteção integral frente ao combate ao trabalho infantil e à regularização do trabalho do adolescente. Revista do Tribunal Superior do Trabalho 2013; 79:55-65.

7. Brasil. Lei no 8.069, de 13 de julho de 1990. Dispõe sobre o Estatuto da Criança e do Adolescente e dá outras providências. Diário Oficial da União 1990; 16 jul.

8. Ramalho HMB, Mesquita SP. Determinantes do trabalho infantil no Brasil urbano: uma análise por dados em painel 2001-2009. Economia Aplicada 2013;17:193-225.

9. Secretaria de Inspeção do Trabalho, Ministério do Trabalho e Emprego. Plano nacional de prevenção e erradicação do trabalho infantil e proteção ao adolescente trabalhador. 2a Ed. Brasília: Ministério do Trabalho e Emprego; 2011.

10. Conselho de Desenvolvimento Econômico e Social. Introdução à proposta do grupo de trabalho aberto para Metas de Desenvolvimento Sustentável Organização das Nações Unidas (ONU). Brasília: Conselho de Desenvolvimento Econômico e Social; 2014.

11. Guimarães JRS. Perfil do trabalho decente no Brasil: um olhar sobre as Unidades da Federação. Brasília: Organização Internacional do Trabalho; 2012.

12. Commission on Social Determinants of Health. Closing the gap in a generation: health equity through action on the social determinants of health. Final report of the Commission on Social Determinants of Health. Geneva: World Health Organization; 2008.

13. Nobre LCC. Trabalho de crianças e adolescentes: os desafios da intersetorialidade e o papel do Sistema Único de Saúde. Ciênc Saúde Coletiva 2003; 8:963-71.
14. Kassouf AL. O que conhecemos sobre o trabalho infantil? Nova Economia 2007; 17:323-50.

15. International Labour Organization. Child labour text book for studants. Geneva: International Labour Organization; 2004.

16. González KA, Pérez RQ, Contreras MY. Determinantes y consecuencias del trabajo infantil: un análisis de la literatura. Revista de la Facultad de Ciencias Económicas: Investigación y Reflexión 2011; 19:113-24.

17. Kassouf AL, McKee M, Mossialos E. Early entrance to the job market and its effect on adult health: evidence from Brazil. Health Policy Plan 2001; 16: 21-8.

18. Ministério da saúde. Trabalho infantil: diretrizes para a atenção integral à saúde de crianças e adolescentes economicamente ativos. Brasília: Ministério da Saúde; 2005. (Série A. Normas e Manuais Técnicos).

19. Nicolella AC, Kassouf AL, Barros ALM. O impacto do trabalho infantil no setor agrícola sobre a saúde. Revista de Economia e Sociologia Rural 2008; 46:673-701.

20. Santos AMA, Tejada CAO, Ewerling F. Os determinantes socioeconômicos do estado de saúde das crianças do Brasil rural. Revista de Economia e Sociologia Rural 2012; 50:473-92.

21. Instituto Brasileiro de Geografia e Estatística. Pesquisa Nacional por Amostra de Domicílios: notas metodológicas. Rio de Janeiro: Instituto Brasileiro de Geografia e Estatística; 2008.

22. Organização Internacional do Trabalho. Eliminar o trabalho doméstico no trabalho infantil e proteger os jovens trabalhadores das condições de trabalho abusivas. Programa Internacional para a Eliminação do Trabalho Infantil (IPEC). Brasília: Organização Internacional do Trabalho; 2012.

23. Organização Internacional do Trabalho. O Brasil sem trabalho infantil! Quando? Projeção de estimativas de erradicação do trabalho infantil, com base em dados de 1992-2003. Brasília: Organização Internacional do Trabalho; 2004.

24. Instituto de Pesquisa Econômica Aplicada. Retratos das desigualdades de gênero e raça. 4a Ed. Brasília: Instituto de Pesquisa Econômica Aplicada; 2011.

25. Kassouf AL. O trabalho de crianças e adolescentes no Nordeste do Brasil. Brasília: Organização Internacional do Trabalho; 2004.

26. Cacciamali MC, Tatei F. Trabalho infantil e o status ocupacional dos pais. Revista de Economia Política 2008; 28:269-90.

27. International Labour Organization; Undertanding Children's Work. Undertanding the Brazilian success in reducing child labour: empirical evidence and policy lessons. Rome: International Labour Organization/Undertanding Children's Work; 2011. 
28. Carvalho IMM. Trabalho infantil no Brasil contemporâneo. Caderno CRH 2008; 21:551-69.

29. Vieira MFA, Matijasevich A, Damiani MF, Madruga SW, Neutzling MB, Menezes AMB, et al. Prevalência de retenção escolar e fatores associados em adolescentes da coorte de nascimentos de 1993 em Pelotas, Brasil. Rev Panam Salud Pública 2012; 31:303-9.

30. Instituto Brasileiro de Geografia e Estatística. Pesquisa Nacional por Amostra de Domicílios: aspectos complementares de educação, afazeres doméstticos e trabalho infantil, PNAD/2006. Rio de Janeiro: Instituto Brasileiro de Geografia e Estatística; 2008.

31. Kassouf AL, Santos MJ. Trabalho infantil no meio rural brasileiro: evidências sobre o "paradoxo da riqueza”. Economia Aplicada 2010; 14:339-53.

32. Lulianelli JA. Violência nas áreas rurais. Crianças e jovens: as principais vítimas. In: Fundo das Nações Unidas para a Infância, organizador. Direitos negados: a violência contra crianças e adolescentes no Brasil. 2a Ed. Brasília: Fundo das Nações Unidas para a Infância; 2006. p. 141-9.

33. Schwartzman S. Trabalho infantil no Brasil. Brasília: Organização Internacional do Trabalho; 2001.

34. Kassouf AL. A ameaça e o perigo à saúde imposto às crianças e jovens em determinados trabalhos. In: Corrêa LB, Vidotti TJ, organizadores. Trabalho infantil e direitos humanos: estudos em homenagem a Oris de Oliveira. Brasília: LTr; 2005. p. 121-45.

35. Organização Internacional do Trabalho. O trabalho infantil na atividade informal urbana em Guarabira - PB: um diagnóstico rápido à luz das piores formas do trabalho infantil. Programa Internacional para Eliminação do Trabalho Infantil (IPEC). Brasília: Organização Internacional do Trabalho; 2006.

36. Organização Internacional do Trabalho. O trabalho doméstico remunerado na América Latina e Caribe. Brasília: Organização Internacional do Trabalho; 2011.
37. International Labour Organization. Children in hazardous work: what we know, what we need to do? Geneva: International Labour Organization; 2011.

38. Facchini LA, Fassa AG, Dall'Agnol M, Maia MFS. Trabalho infantil em Pelotas: perfil ocupacional e contribuição à economia. Ciênc Saúde Coletiva 2003; 8:953-61.

39. Fassa AG, Facchini LA, Dall'Agnol MM, Christiani DC. Child labor and musculoskeletal disorders: the Pelotas (Brazil) epidemiological survey. Public Health Rep 2005; 120:665.

40. Benvegnú LA, Fassa AG, Facchini LA, Wegman DH, Dall'Agnol MM. Work and behavioural problems in children and adolescents. Int J Epidemiol 2005; 34:1417-24.

41. Giatti L, Campos MO, Crespo CD, Andrade SSCA, Barreto SM. Labor in early life, vulnerability for health in Brazilian schoolchildren: National Adolescent School-based Health Survey (PeNSE 2012). Rev Bras Epidemiol 2014; 17:17-30.

42. Fischer FM, Oliveira DC, Teixeira LR, Teixeira MC TV, Amaral MA. Efeitos do trabalho sobre a saúde de adolescentes. Ciênc Saúde Coletiva 2003; 8: 973-84.

43. Jardim R, Barreto SM, Giatti L. Confiabilidade das informações obtidas de informante secundário em inquéritos de saúde. Cad Saúde Pública 2010; 26:1537-48.

44. Barros MBA, Zanchetta LM, Moura EC, Malta DC Autoavaliação da saúde e fatores associados, Brasil, 2006. Rev Saúde Pública 2009; 43:27-37.

45. Cacciamali MC, Tatei F, Batista NF. Impactos do programa bolsa família federal sobre o trabalho infantil e a frequência escolar. Revista de Economia Contemporânea 2010; 14:269-301.

Recebido em 19/Set/2014

Versão final reapresentada em 05/Abr/2015

Aprovado em 04/Mai/2015 